\title{
Silica nanodisks as platforms for fluorescence lifetime-based sensing of $\mathbf{p H}$
}

\author{
SUBHASREE BANERJEE, ANJALI DHIR, TUSEETA BANERJEE, \\ AVINASH KUMAR SINGH and ANINDYA DATTA* \\ Department of Chemistry, Indian Institute of Technology Bombay, Powai, Mumbai 400 076, India \\ e-mail: anindya@chem.iitb.ac.in
}

\begin{abstract}
Core-shell conjugates of silica nanodisks and fluorescent dyes have been prepared. Rhodamine $\mathrm{B}$, the reference, has been attached to the core, by surface functionalization of the pristine SNDs. Then, a layer of silica has been deposited on the composite nanodisks. Finally, the surface has been functionalized with fluorescein in one case and protoporphyrin IX in the other. These dyes exhibit $\mathrm{pH}$-dependent fluorescence properties. The nanoconjugates are found to sense the $\mathrm{pH}$ of the medium, through systematic variation of the fluorescence intensity ratios of the reporter dye at the surface and the reference dye at the core. Moreover, the fluorescence lifetimes and corresponding amplitudes of the reporter dyes have been found to be reliable parameters for assessing the $\mathrm{pH}$ of the medium, even though the variation in lifetimes of fluorescein is rather small. In case of protoporphyrin, however, this variation is significantly large. Besides, the change in amplitudes is prominent in acidic as well as alkaline solutions. The temporal parameters can thus be used to ascertain the $\mathrm{pH}$ of the medium, when used in conjunction with each other.
\end{abstract}

Keywords. Fluorescence; sensor; silica; nanoparticle; lifetime.

\section{Introduction}

Fluorescence sensing has grown into a popular, widely used methodology in diagnostics and cellular imaging. ${ }^{1}$ Highly luminescent and photostable biolabelling agents are required in this technique. ${ }^{2-4}$ Organic fluorophores are the most commonly used fluorescent sensors due to the sensitivity of their fluorescent properties to subtle changes in microenvironments. ${ }^{5}$ However, constraints like photodegradation and false positives often encumber this otherwise sensitive technique. Moreover, the fluorescence intensity of organic fluorophores depends on the concentration of both analyte and the sensor. ${ }^{6}$ Quatum dots may be used to circumvent these problems to a large extent, but their use is restricted by their toxicity towards cells. ${ }^{7}$ Entrapment of fluorescent organic molecules inside small particles of polymer, silica and lipid assemblies shields the molecule from several environmental factors like oxygen and causes amplification of the signal. ${ }^{8-11}$ The quantitative measurement of the analyte concentration becomes easy as the fluorescence intensity of the doped sensor molecule becomes independent of the concentration of the sensor molecule.

\footnotetext{
${ }^{*}$ For correspondence
}

Several reports exist on the encapsulation of fluorescent dyes in silica nanostructures. The popularity of this matrix stems from the facile surface functionalization by simple silanol chemistry. ${ }^{12}$ Large surfaceto-volume ratio of the nanostructures facilitates their binding to biomolecules in large numbers. Besides, silica nanostructures are non-toxic, ${ }^{13}$ chemically inert and optically transparent. ${ }^{14}$ Because of these advantages, nanoconjugates of silica and fluorescent dyes have generated significant interest in the field of sensing of analytes in microheterogeneous media. ${ }^{15} \mathrm{~A}$ brief discussion of some of the recent work in this area has been presented in the next paragraph.

Kopelman and co-workers have designed fluorescence ratiometric sensors of intracellular oxygen, based on conjugates of dyes with silica nanoparticles. ${ }^{11}$ More recently, Weisner and co-workers have used a modified Stöber's method to prepare core-shell dye-silica nanoconjugates. ${ }^{16-18}$ This architecture has enabled the dye molecules to get decoupled from each other. With this background, core-shell nanoparticles have been prepared for sensing $\mathrm{pH} .{ }^{19}$ In these sensors, trimethylrhodamine isothiocyanate (TRITC) and the fluorescein isothiocyanate (FITC) have been used to functionalize the core and the shell, respectively, so that the reporter dye, fluorescein, is exposed to the external $\mathrm{pH}$ while the reference dye, rhodamine $\mathrm{B}$, is sequestered from 
the medium whose $\mathrm{pH}$ is to be measured. The ratio of the fluorescence intensity of the reporter and the reference dyes has been used as the metric to determine the $\mathrm{pH}$ of the medium. Such ratiometric measurement obviates interference from factors like excitation source fluctuations and sensor concentrations.

In the present work, we have adopted a similar approach as Weisner and co-workers, but have used anisotropic silica nanodisks prepared using lamellar micelles as templates. ${ }^{20-22}$ These nanodisks are likely to have important applications in drug delivery and sensing in biological media, as Mitragotri and co-workers have shown that anisotropic structures are less prone to phagocytosis by macrophages and consequent ejection from the body. ${ }^{23,24}$

Further, we have used these core-shell nanoconjugates as a platform to explore the feasibility of $\mathrm{pH}$ sensing with the fluorescence lifetimes, in addition to the ratios of intensities. In doing so, a different reporter dye, protoporphyrin IX (PP), has been found to bear a great promise than fluorescein. In neat aqueous solutions, protoporphyrin exhibits $\mathrm{pH}$ dependent equilibria involving its monomer and aggregates, at different degrees of protonation. These species are characterized by different fluorescence quantum yields and emission maxima. ${ }^{25}$ In the $\mathrm{pH}$ range of $10-12$, strong fluorescence is observed, with the spectral maximum at $620 \mathrm{~nm}$. This is ascribed to deprotonated aggregates (figure 2 of reference 25). At $\mathrm{pH}=6$, fluorescence intensity becomes negligible due to protonation of carboxylic groups of protoporphyrin and formation of larger aggregates. The fluorescence maximum is reported to shift to $634 \mathrm{~nm}$ for $\mathrm{pH} 3-6 .{ }^{25}$ In the narrow $\mathrm{pH}$ range of $1-2$, the free base porphyrins get protonated to form dications. The fluorescence maximum for these dications are observed at $606 \mathrm{~nm}$. The present work seeks to exploit this remarkable $\mathrm{pH}$ dependence of protoporphyrin in designing an efficient fluorescence sensor of $\mathrm{pH}$.

\section{Experimental}

Silica nanodisks (SNDs) have been prepared using lamellar micelles as templates, as has been described earlier (figure S1). ${ }^{20,21}$ The surface of these nanodisks have been functionalized with rhodamine $\mathrm{B}$ by the procedure described in the next few lines. All these steps have been performed in a nitrogen atmosphere and in the dark. A mixture of $13 \mathrm{mg}$ of Rhodamine B isothiocyanate, RITC (Aldrich, India) and $100 \mu \mathrm{L}$ 3-Aminopropyltrimethoxysila- ne, APTMS has been added to $30 \mathrm{~mL}$ anhydrous ethanol. This mixture is stirred for $24 \mathrm{~h}$ to obtain the RITC-APTMS conjugate. $20 \mathrm{~mL}$ of this conjugate, $100 \mu \mathrm{L}$ tetraethyl orthosilicate, TEOS (Aldrich, India) and $3 \mu \mathrm{L}$ Triethylamine $\left(\mathrm{Et}_{3} \mathrm{~N}\right)$ (Spectrochem, India) are added to $150 \mathrm{mg}$ silica nanodisk and stirred for $24 \mathrm{~h}$. Then, $100 \mu \mathrm{L}$ TEOS is added and the mixture is stirred further for $24 \mathrm{~h}$, in order to deposit a layer of silica between the core and the shell. Next, the surface of this nanocomposite has been functionalized with the reporter dye, fluorescein or protoporphyrin IX. In order to perform functionalization with fluorescein, $8 \mathrm{mg}$ of Fluorescein isothiocyanate, FITC (Aldrich, India) and $100 \mu \mathrm{L}$ of APTMS (Aldrich, India) are stirred in $30 \mathrm{ml}$ ethanol for $24 \mathrm{~h} .20 \mathrm{~mL}$ of this mixture is then added to the rhodamine-functionalized nanodisk suspension and stirred for $24 \mathrm{~h}$ more. Core-shell nanodisks are then obtained by multiple centrifugation and washing with ethanol and water (scheme 1). Such coreshell nanodisks are referred to as RITC/FITC SNDs. In order to functionalize the surface of rhodaminelabelled nanodisks by protoporphyrin IX, a mixture of $13 \mathrm{mg}$ Protoporphyrin IX, PP (Aldrich, India), $100 \mu \mathrm{L}$ APTMS, $3 \mathrm{mg}$ Hydroxybenzotriazole, HOBT (Spectrochem, India), $5 \mathrm{mg} \mathrm{N}^{\prime}$-dicyclohexylcarbodiimide, DCC (Aldrich, India) and $4 \mu \mathrm{L} \mathrm{Et}_{3} \mathrm{~N}$ in $30 \mathrm{~mL}$ anhydrous ethanol is stirred for $24 \mathrm{~h} .20 \mathrm{~mL}$ of this PP-APTMS conjugate is used to prepare the shell of rhodamine B - functionalized SND core in the similar fashion as mentioned for RITC/FITC SNDs (scheme 1). This type of nanodisk is referred to as RITC/PP SNDs.

The SEM images have been obtained on a scanning electron microscope (SEM, HITACHI, S-3400 N). Steady state spectra have been recorded with JASCO V530 Spectrophotometer and Varian Cary Eclipse Fluorimeter. Time resolved fluorescence studies have been performed at magic angle polarization in a time correlated single photon counting (TCSPC) system, from IBH, UK, with $\lambda_{e x}=406 \mathrm{~nm}$. The full width at half maximum of the instrument response function is $250 \mathrm{ps}$. The PL decays are fitted by an iterative reconvolution method using IBH DAS 6.2 software, to the biexponential function $\left(I(t)=I(0)\left[A_{1} \tau_{1}+A_{2} \tau_{2}\right]\right.$, where $I(t), I(0)$ are the fluorescence intensities at time $t$ and zero, respectively, after instantaneous excitation, $\tau_{1}, \tau_{2}$ are the two lifetimes and $A_{1}, A_{2}$ are the corresponding amplitudes, such that $\left.A_{1}+A_{2}=1\right){ }^{26,27} \mathrm{~A}$ global analysis of the data has been used, as discussed later. In both the steady state and time resolved studies, the external $\mathrm{pH}$ was maintained at the desired values by using appropriate buffer solutions. ${ }^{28}$ 

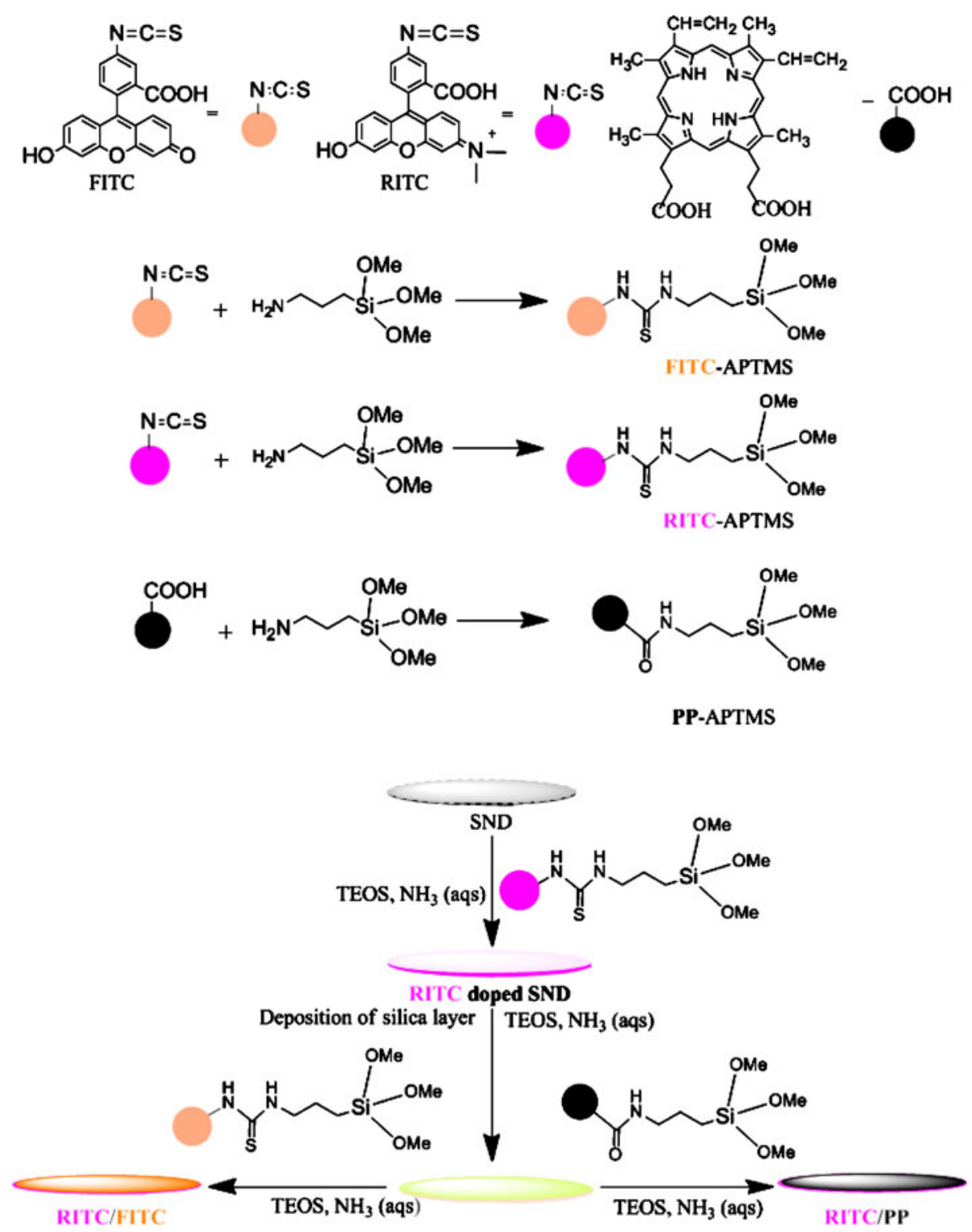

Scheme 1. Preparation of dual dye doped silica nanodisk, where RITC is doped on SND by the surface of modification. This surface modified nanodisk act as internal standard (magenta). There is a layer of RITC sequestered silica at the outer surface of SND (orange). This layer acts as the sensor layer. Both the reference and the sensor layer are separated by a layer of silica (yellow).

\section{Results and discussion}

Absorption spectra are shown in figure S2. The steady state fluorescence spectra obtained from the RITC/FITC SNDs are normalized to have the same value of intensity at $580 \mathrm{~nm}$, which is the fluorescence maximum of rhodamine $B$. In these normalized spectra, the fluorescence spectrum characteristic of fluorescein, with a maximum at $517 \mathrm{~nm}$, is found to become stronger upon changing the external $\mathrm{pH}$ from 5.0 to 8.8 (figure 1a). An excitation wavelength of $480 \mathrm{~nm}$ is used, so as to ensure the excitation of the sensor as well as the reference dye. The increase of the fluorescence intensity of fluorescein with respect to rhodamine B indicates the formation of dianions $(\phi=0.92)^{29}$ at the cost of monoanions $(\phi=0.36)^{29}$. The plot of the ratios of fluorescence intensities of fluorescein $\left(\lambda_{\mathrm{em}}=\right.$ $517 \mathrm{~nm})$ and rhodamine $\mathrm{B}\left(\lambda_{e m}=580 \mathrm{~nm}\right)$ exhibits a sigmoidal nature, with a point of inflection at $\mathrm{pH}=$ 6.3 (figure 2a). This observation agrees with the model involving a two-state equilibrium between monoanionic and dianionic forms of FITC existing in this $\mathrm{pH}$ range. 


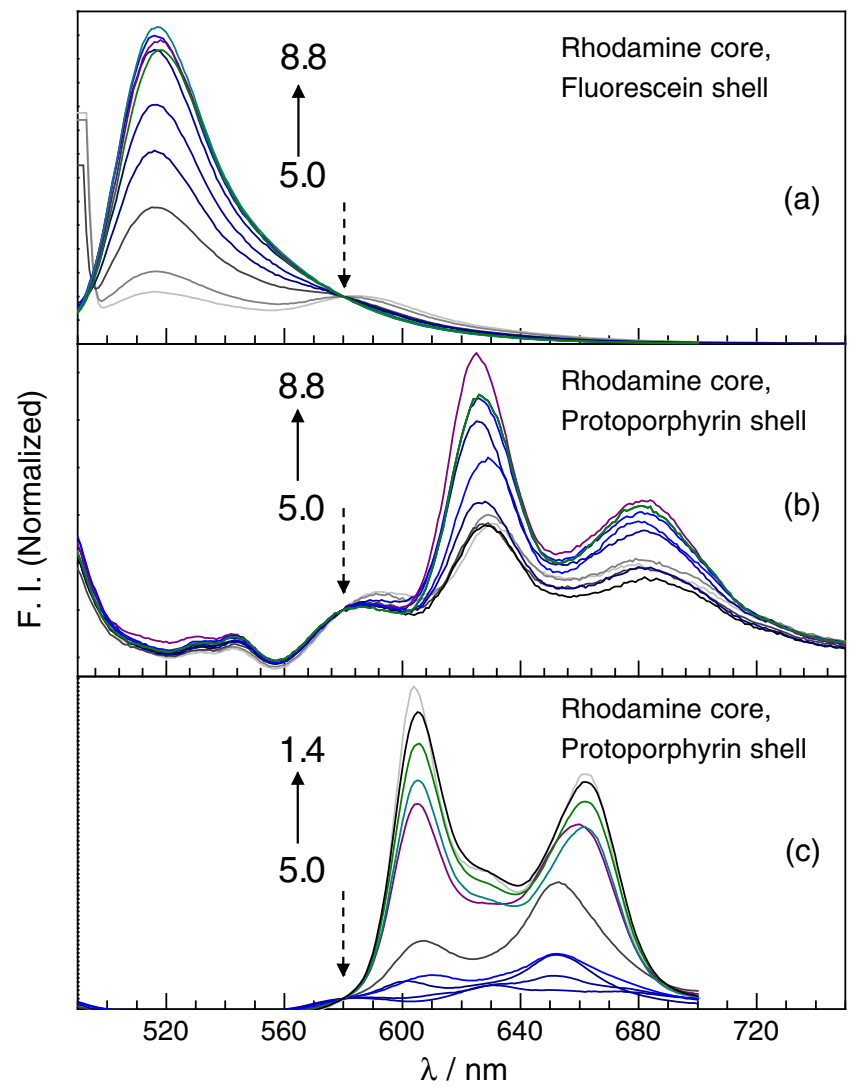

Figure 1. Flurescence spectra of (a) RITC/FITC SNDs in buffers of $\mathrm{pH}=5.0,5.4,5.8,6.2,6.6,7.0,7.8,8.4,8.6$ and 8.8. $\lambda_{\text {ex }}=480 \mathrm{~nm}$. (b) RITC/PP SNDs in buffers of $\mathrm{pH}=$ 5.0, 5.4, 5.8, 6.2, 6.6, 7.2, 7.8, 8.0, 8.2, 8.4, 8.6 and $8.8 \lambda_{\mathrm{ex}}=$ $406 \mathrm{~nm}$. (c) RITC/PP SNDs in buffers of $\mathrm{pH}=1.4,1.6,1.8$, $2.0,2.2,2.6,3.4,4.0,4.4$ and 5.0. $\lambda_{\mathrm{ex}}=406 \mathrm{~nm}$. The spectra are normalized at the fluorescence maximum of rhodamine $\mathrm{B}(580 \mathrm{~nm}$, indicated by dashed arrows in the spectra).

The trend is the same as reported earlier RITC/ FITC nanoparticles. ${ }^{19,29}$

Next, we explore the feasibility of using fluorescence lifetimes for $\mathrm{pH}$ sensing with the RITC/ FITC SNDs, as lifetimes are often believed to be superior parameters for sensing, even though their measurement is significantly more difficult. ${ }^{30-33}$ For these studies, the decays have been collected at $\lambda_{e m}=517 \mathrm{~nm}$ (dominated by fluorescein) and $580 \mathrm{~nm}$ (dominated by rhodamine B). The fluorescence decays have been found to become progressively slower, with increase in the $\mathrm{pH}$ of the medium (figure S3b). This trend is in line with the expectation, as the lifetime of fluorescein increases from $3.3 \mathrm{~ns}$ to $3.9 \mathrm{~ns}$ upon increasing the $\mathrm{pH}$ from 6 to 9 in neat aqueous solutions. ${ }^{34}$ The slowing down of the decays at $517 \mathrm{~nm}$ is attributed to fluorescein as well, as it has a significant fluorescence intensity at this emission wavelength. The lifetime of rhodamine $\mathrm{B}$ is not
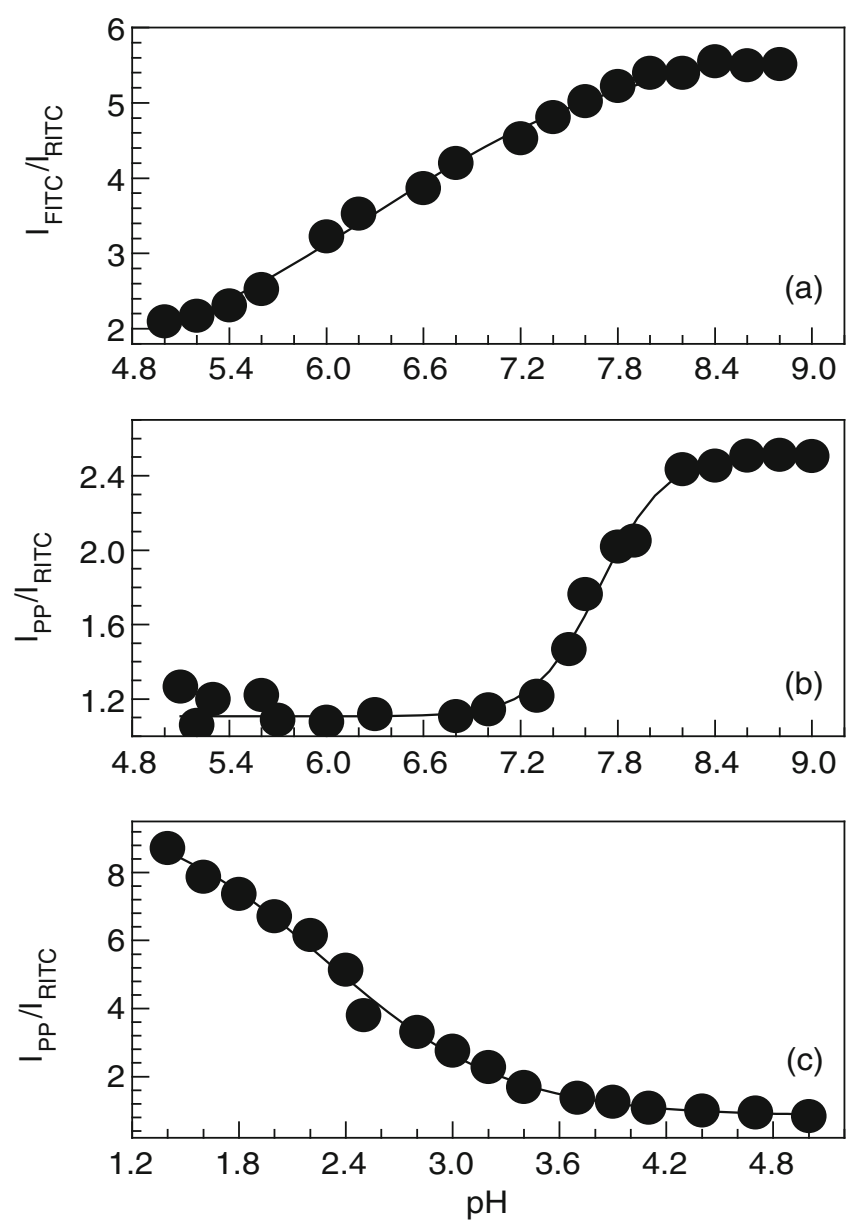

Figure 2. Ratiometric calibration curves for (a) RITC/FITC SNDs in buffer solutions, $\mathrm{pH}=5.0 \leq \mathrm{pH} \leq 8.8$. The ratios of intensity at $517 \mathrm{~nm}$ (fluorescence maximum of FITC) to that at $580 \mathrm{~nm}$ (fluorescence maximum of RITC) have been plotted; (b) and (c) ratiometric calibration curves for RITC/PP SNDs in buffer solutions. (b) $5.0 \leq \mathrm{pH} \leq 9.0$, Probe wavelength $=603 \mathrm{~nm}$ and (c) $1.4 \leq \mathrm{pH} \leq 5.0$. Probe wavelength $=630 \mathrm{~nm}$.

expected to vary, as it is sequestered in the core. With these considerations, global analysis ${ }^{35-37}$ of the temporal data has been performed on sets of two decays, at the same $\mathrm{pH}$ but at two different emission wavelengths. The lifetimes have been set as the global parameters while the pre-exponential factors are treated as local parameters. It is observed that the longer global lifetime $\left(\tau_{1}\right)$ increases in a sigmoidal manner with increase in $\mathrm{pH}$ (figure 3, tables $\mathrm{S} 1$ and $\mathrm{S} 2$ ), from $3.4 \mathrm{~ns}$ at $\mathrm{pH}=5$ to $3.8 \mathrm{~ns}$ at $\mathrm{pH} 8.8$, while the shorter lifetime remains fixed at $\sim 1.6 \mathrm{~ns}$. These two sets of values match the lifetimes of fluorescein and rhodamine $\mathrm{B}^{38}$, respectively and are thus assigned. The improvement obtained using the temporal parameters, over the steady state spectral parameters, is that the lifetimes of the two emitting species are well-defined and do not interfere with each 


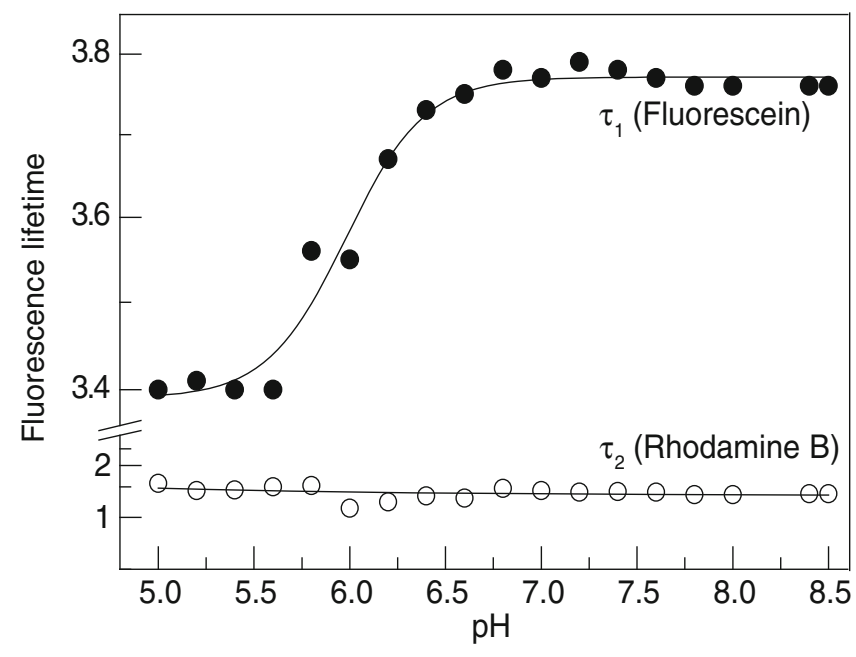

Figure 3. Variation of $\tau_{1}$ (filled circle) and $\tau_{2}$ (hollow circle) for RITC/FITC-doped SNDs at different $\mathrm{pH}$.

other. The shortcoming of the present system, however, is that the variation of the lifetime of the marker dye is very little. It is not realistic to expect to be able to perform $\mathrm{pH}$ sensing with a good level of sensitivity, with such a small variation in the fluorescence lifetimes. This problem can be overcome by replacing the marker dye with another one, which exhibits a more prominent variation in lifetime, as a function of $\mathrm{pH}$. This is the objective for studying silica nanodisks in which the surface is functionalized by protoporphyrin instead of fluorescein. The choice of protoporphyrin as the sensing fluorophore is inspired by the fact that in neat aqueous solutions, its fluorescence lifetime exhibits a larger change, from $\sim 6 \mathrm{~ns}$ in acidic solution to $\sim 12 \mathrm{~ns}$ in alkaline ones (figure $\mathrm{S} 4$ ). The protoporphyrin at the surface of the RITC/PP SNDs exhibits an interesting feature. A significant fluorescence intensity is observed at $630 \mathrm{~nm}$, even when the external $\mathrm{pH}$ is close to $5-6$, in contrast to the observation in neat aqueous solutions. This is likely to be so because the fluorophores are in a less aggregated state, when they bind to the SNDs. Nevertheless, the fluorescence intensity is at a minimum at this $\mathrm{pH}$. The spectra, normalized to the rhodamine $\mathrm{B}$ fluorescence, become progressively more intense upon increasing the $\mathrm{pH}$ up to 12 (figure $1 \mathrm{~b}$ ). The fluorescence maximum changes very little in the alkaline solution and may be assigned to the deprotonated form of protoporphyrin. Upon decreasing the $\mathrm{pH}$, a more marked increase in intensity is observed, but this is associated with a pronounced change in the spectral shape (figure 1c). The spectrum gets blue shifted and a new emission band develops at $605 \mathrm{~nm}$, which corres- ponds to the emission band of the protonated species of protoporphyrin .

Plots of the variation of the protoporphyrin emission intensities at the spectral maxima, with the rhodamine $\mathrm{B}$ intensities normalized to the same value, are sigmoidal for both the $\mathrm{pH}$ ranges (5.0-11.5 and 5.0-1.4) (figure $2 \mathrm{~b}, \mathrm{c}$ ). The inflection points occur at $\mathrm{pH}=7.7$ and 2.2. Thus, RITC/PP SNDs are found to act as $\mathrm{pH}$ sensors, much like the RITC/FITC SNDs, with the added advantage that the $\mathrm{pH}$ of acidic solutions can also be sensed using these core-shell nanostructures, using a combination of the ratio of the fluorescence intensities of protoporphyrin and rhodamine $\mathrm{B}$, with the fluorescence maximum of protoporphyrin.

The last question to be addressed in the present study concerns the feasibility of using RITC/ PP SNDs in fluorescence lifetime-based $\mathrm{pH}$ sensing. With a change in the external $\mathrm{pH}$, the fluorescence decays are found to become faster in the range of $\mathrm{pH}=1.9$ to 5.0 and then slower in the range of $\mathrm{pH}=5-11.5$, at $\lambda_{\mathrm{em}}$ $=580 \mathrm{~nm}$ (figure S4) as well as $605 \mathrm{~nm}$ (figure $\mathrm{S} 6$, upper panel) and $630 \mathrm{~nm}$ (figure S6, lower panel). This trend is in agreement with that observed in the variation of normalized fluorescence intensities (figure 1b, c). Global analysis has been performed on these decays, in the same manner as has been adopted for RITC/FITC SNDs. The longer lifetime exhibits a sigmoidal increase with $\mathrm{pH}$, similar in nature to the case of RITC/FITC SNDs (figure 4a). An inflection point is obtained at $\mathrm{pH}=6.1$ and the region of $\mathrm{pH}$ over which the lifetime changes is 4.4-8.0. A quantitiative improvement in these experiments is that the lifetime changes from $6.5 \mathrm{~ns}$ to ca. $14 \mathrm{~ns}$, which is a significantly larger variation than that observed for fluorescein. So, the protoporphyrin-labelled nanodisks bear the promise of being more sensitive fluorescence lifetime-based sensors of $\mathrm{pH}$ in the range of 4.4-8.0. This range is larger than that covered by the variation of the fluorescence intensity of fluorescein and this is likely to render the protoporphyrin-labelled silica nanodisks a useful sensor in situations where the $\mathrm{pH}$ is abnormally lower than the physiological $\mathrm{pH}$, like in the case of the extracellular $\mathrm{pH}$ in tumour tissues, which is reported to be as low as 5.0-5.5. ${ }^{39}$ This $\mathrm{pH}$ region cannot be explored by protoporphyrin by itself, as it forms aggregates with very little, if any, fluorescence in this region. Binding with the silica nanostructure disrupts these aggregates to a large extent and thus, makes it possible for protoporphyrin to function in this region of acidity.

At this point, it might appear that the reference fluorophore, rhodamine $\mathrm{B}$, has no role to play in 


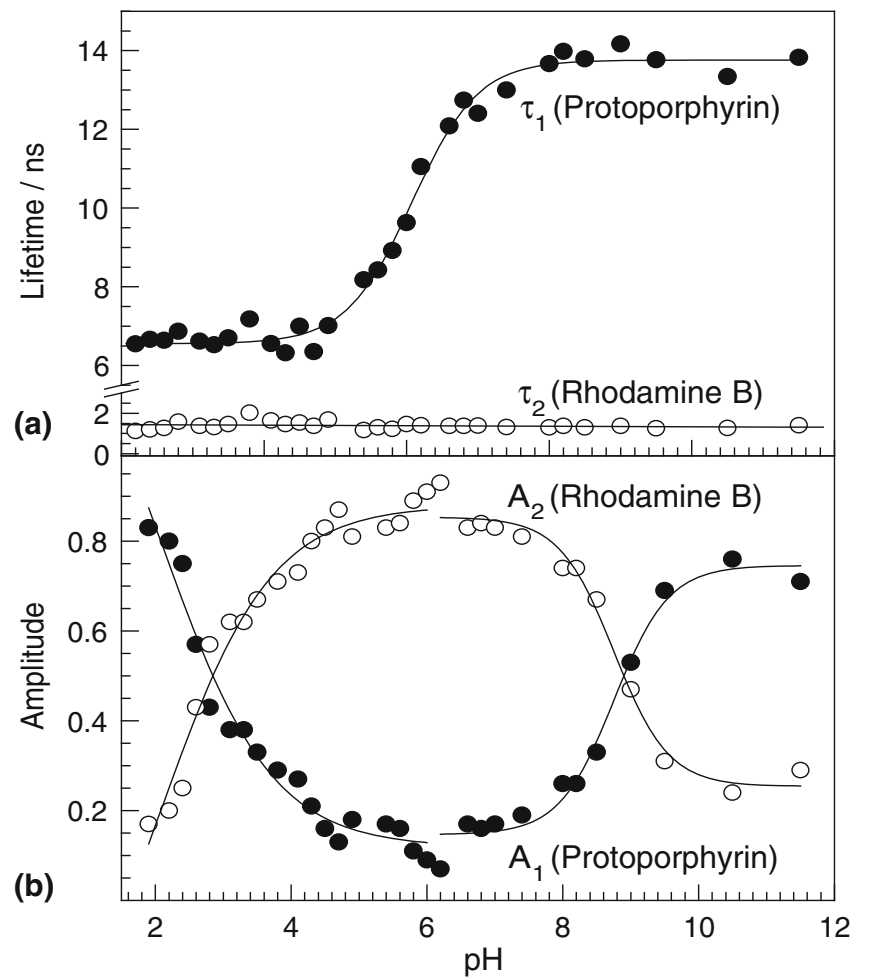

Figure 4. Variation of (a) fluorescence lifetimes and (b) amplitudes for RITC/PP SNDs at different $\mathrm{pH}$.

lifetime-based sensing and that it would be sufficient to use nanodisks whose surfaces are labelled with protoporphyrin. However, the reference dye, at the core, proves to be useful in extending the range of operation of the $\mathrm{pH}$ sensor further to more acidic conditions. This is achieved by considering the variation of the amplitudes, along with that of the lifetimes. The amplitude associated with the fluorescence of protoporphyrin exhibits a minimum at $\mathrm{pH}=6.0$ and a sigmoidal variation for increase as well as decrease of $\mathrm{pH}$ (figure $4 \mathrm{~b}$ ). This is because of the amplitude denotes the relative contribution of the fluorophore to the total fluorescence, with respect to the reference dye, rhodamine B. The lifetime and quantum yield of rhodamine $\mathrm{B}$ remains constant over the entire range of $\mathrm{pH}$, as it is sequestered in the core. However, it contributes to the total fluorescence in a greater extent in conditions for which the fluorescence intensity of protoporphyrin is small. This is why its amplitude decreases while that of protoporphyrin increases when the external $\mathrm{pH}$ is changed either way from the value of 5-6, for which protoporphyrin is least emissive. This is what causes the variation in the amplitudes and provides an additional parameter for $\mathrm{pH}$ sensing, besides steady state intensity and fluorescence lifetime. It should be remembered that the amplitudes are not single-valued functions of the $\mathrm{pH}$ and so, cannot be used by themselves to ascertain the correct $\mathrm{pH}$ of the medium. However, they are obtained in the same measurement that yields the lifetimes as well. When used in conjunction with the lifetimes, the amplitudes emerge to be useful metrics of determination of $\mathrm{pH}$, especially for acidic solutions.

\section{Conclusion}

Core-shell nanoconjugates of SNDs and organic fluorophores have been synthesized, where rhodamine B resides in the core and fluorescein or protoporphyrin resides on the surface of SNDs. Steady state fluorescence intensity and lifetimes of the reporter dyes in these nanoconjugates show variation with the external $\mathrm{pH}$. Therefore, these nanoconjugates may be useful for sensing the $\mathrm{pH}$ of the microenvironments. In case of RITC/FITC SNDs, the variation of fluorescence intensity of fluorescein $\left(\lambda_{\mathrm{em}}=517 \mathrm{~nm}\right)$, normalized with respect to that of, rhodamine $\mathrm{B}\left(\lambda_{\mathrm{em}}=\right.$ $580 \mathrm{~nm}$ ), is sigmoidal for $\mathrm{pH}=5.5-8.0$. Fluorescence lifetimes at emission wavelength of $517 \mathrm{~nm}$ show a sigmoidal variation as well, but the change in lifetimes is rather small. This has been the motivation to prepare RITC/PP SNDs, in which protoporphyrin acts as the sensor dye. The normalized fluorescence intensities of protoporphyrin in these nanoconjugates, when used in conjuction with the positions of the fluorescence maxima, can sense $\mathrm{pH}$ in the alkaline as well as acidic solutions. Besides, the lifetime of protoporphyrin varies from $6 \mathrm{~ns}$ to ca. $14 \mathrm{~ns}$, upon increasing the external $\mathrm{pH}$. The amplitudes vary with $\mathrm{pH}$ as well, being minimum at $\mathrm{pH}=5-6$ and increasing for higher as well as lower $\mathrm{pH}$. Thus, the temporal parameters of protoporphyrin in these nanoconjugates can also be used to sense the $\mathrm{pH}$ of the medium.

\section{Supplementary information}

The supplementary information of additional steady state and time resolved date are provided in figure S1S6, tables S1-S2 (see www.ias.ac.in/chemsci).

\section{Acknowledgements}

This work was supported by Council of Scientific and Industrial Research (CSIR) grant to AD. SB, AD and AKS thank (CSIR) for research fellowships. Department of MEMS and CRNTS, IIT Bombay are thanked for electron microscopy facilities. The authors are indebted to Dr. S L Kamath for his guidance with SEM. 


\section{References}

1. Lakowicz J R 1999 Principles of fluorescence spectroscopy, 2nd ed. New York, Kluwer Academic

2. Sharma P, Brown S, Walter G, Santra S and Moudgil B 2006 Adv. Coll. Interface. Chem. 123471

3. Medintz I L, Tetsuo U H, Goldman E R and Mattoussi H 2005 Nat. Mater. 4435

4. Michalet X, Pinaud F F, Bentolila L A, Tsay J M, Doose S and Li J J 2005 Science 307538

5. Zhang J, Campbell R E, Ting A Y and Tsien R Y 2002 Nat. Rev. 3906

6. Gryczynski Z, Gryczynski I and Lakowicz J R 2003 Methods Enzymol. 36044

7. Wang L, Zhao W and Tan W 2008 Nano Res. 199

8. Buck S M, Koo Y -E L, Park E, Xu H, Philbert M A, Brasuel M A and Kopelman R 2004 Curr. Opin. Chem. Biol. 8540

9. Nguyen T and Rosenzweig Z 2002 Anal. Bioanal. Chem. 37469

10. Ma A and Rosenzweig Z 2005 Anal. Bioanal. Chem. 38228

11. Xu H, Aylott J W, Kopelman R, Miller T J and Philbert M A 2001 Anal. Chem. $\mathbf{7 3} 4124$

12. Zhong W 2009 Anal. Bioanal. Chem. 39447

13. Baca H K, Ashley C, Carnes E, Lopez D, Flemming J, Dunphy D, Singh S, Chen Z, Liu N, Fan H, Lopez G P, Brozik S M, Washburne M W- and Brinker C J 2006 Science $\mathbf{3 1 3} 337$

14. Kao W and Hunt A J 1994 J. Non-Cryst. Solids 17618

15. Ro H and Carson J H 2004 J. Biol. Chem. 27937115

16. Ow H, Larson D R, Srivastava M, Baird B A, Webb W and Wiesner U 2005 Nano Lett. 5113

17. Stöber W, Fink A and Bohn E 1968 J. Colloid Interface Sci. 2662

18. Bogush G H, Tracy M A and Zukoski C F 1988 J. NonCryst. Solids 10495

19. Burns A, Sengupta P, Zedayko T, Baird B and Wiesner U 2006 Small 26723

20. Banerjee S and Datta A 2010 Langmuir 261172
21. Banerjee S, Ghosh H and Datta A 2011 J. Phys. Chem. C 11519023

22. Banerjee S, Honkote S and Datta A 2011 J. Phys. Chem. C 1151576

23. Mitragotri S and Lahann J 2008 Natl. Mater 815

24. Champion J A and Mitragotri S 2006 Proc. Natl. Acad. Sci. USA 1034930

25. Melø T B and Reisæter G 1986 Biophys. Chem. 2599

26. Patel S and Datta A 2007 J. Phys. Chem. B 111 10557

27. Panda D, Khatua S and Datta A 2007 J. Phys. Chem. B 1111648

28. Robinson R A and Stokes R H 1968 Electrolyte solutions, 2nd ed. London, Butterworths

29. Haugland R P 2005 The handbook-A guide to fluorescent probes and labeling technologies, 10th ed. Eugene OR USA, Molecular Probes

30. Saxl T, Khan F, Ferla M, Birch D J S and Pickup J 2011 Analyst 136968

31. Ogikubo $\mathrm{S}$, Nakabayashi $\mathrm{T}$, Adachi $\mathrm{T}$, Islam $\mathrm{M} \mathrm{S}$, Yoshizawa T, Kinjo M and Ohta N 2011 J. Phys. Chem. B 11510385

32. Zhang J, Fu Y and Lakowicz J R 2011 J. Phys. Chem. C 1157255

33. Stringari $\mathrm{C}$, Cinquin A, Cinquin O, Digman $\mathrm{M}$ A, Donovan P J and Gratton E 2011 Proc. Natl. Acad. Sci. USA 10813582

34. Liu B, Fletcher S, Avadisian M, Gunning $\mathrm{P} T$ and Gradinaru C C 2009 J. Fluoresc. 19915

35. Boens N and De Schryver F C 2006 Chem. Phys. 325 461

36. Dommelen L V, Boens N, De Schryver F C and Ameloot M 1995 J. Phys. Chem. 998959

37. Chowdhury P K, Halder M, Sanders L, Calhoun T, Anderson J L, Armstrong D W, Song X and Petrich J W 2004 J. Phys. Chem. B 10810245

38. Magde D, Rojas G E and Seybold P 1999 Photochem. Photobiol. 70737

39. Newell K, Franchi A, Pouyssegur J and Tannock I 1993 Proc. Natl. Acad. Sci. USA 901127 